\title{
Ectoparasitic Infestations
}

\author{
Jörg Heukelbach, MD, PhD*, ShelleyF. Walton, BSc, PhD, \\ and Hermann Feldmeier, MD, PhD
}

\begin{abstract}
Address
*Departamento de Saúde Comunitária, Faculdade de Medicina, Universidade Federal do Ceará, Rua Prof. Costa Mendes 1608, $5^{\circ}$ andar, Fortaleza CE 60430-I40, Brazil.

E-mail: samsa@mcanet.com.br
\end{abstract}

Current Infectious Disease Reports 2005, 7:373-380

Current Science Inc. ISSN 1523-3847

Copyright () 2005 by Current Science Inc.

Ectoparasitic infestations are highly prevalent in resourcepoor populations throughout the world and are associated with considerable morbidity. Reliable data on the epidemiology, immunology, and therapy of ectoparasitic infestations and on the biology of the parasites remain scarce. The control of parasitic skin diseases, such as scabies, pediculosis, tungiasis, myiasis, and cutaneous larva migrans in endemic areas remains a challenge. Using appropriate study designs, it is imperative to increase further the knowledge on the various aspects of the parasites and the infestations they cause.

\section{Introduction}

Ectoparasitic infestations such as scabies, pediculosis, cutaneous larva migrans, tungiasis, and myiasis are prevalent, but neglected diseases. They pose a serious health risk in resource-poor communities, particularly in the developing world, and are associated with considerable morbidity.

\section{Scabies}

The burrowing ectoparasitic mite Sarcoptes scabiei is regarded as an important public health problem worldwide, especially for developing countries, indigenous populations, and immunocompromised individuals. It is also a significant veterinary and wildlife disease. Classical complications include secondary infection with Staphylococcus aureus and group A streptococcus and the associated sequelae of cellulitis, lymphangitis, and acute glomerulonephritis. Scabies has been a recognized disease for at least 3000 years and described in ancient China, India, and the Middle East. It is transmitted by contact and, therefore, is a disease of overcrowding and poverty rather than reflecting low hygiene. Scabies and pyoderma have recently been identified as contributing to the multideterminant risk factors in excessive renal failure and other chronic morbidities in Australian Aborigines [1].

\section{Clinical presentation and immunodiagnosis}

In humans, the clinical signs and symptoms of scabies can mimic other skin diseases, such as eczema, impetigo, tinea, allergic reactions, and contact dermatitis, consequently making diagnosis problematic.

Clinical presentation with a primary infestation of scabies is reported to take 4 to 6 weeks to develop after infection, and often individuals have already transmitted the disease to others prior to receiving therapy. Currently, definitive diagnosis is via clinical signs and microscopic examination of skin scrapings, but experience has shown the sensitivity of these traditional tests is less than $50 \%$. Many studies document that scabies-mite infestation causes the production of measurable antibodies in infested hosts [2]. Enzyme-linked immunosorbent assays have been developed for the detection of antibodies to S. scabiei in pigs and dogs. These assays rely on whole-mite antigen preparations derived from $S$. scabiei suis, and the itch-mite of the red fox S. scabiei vulpes [3]. A recent study, looking at cross-reacting IgG antibodies to the fox-mite antigen extract in human scabies, reported a sensitivity of only $48 \%$, compared with $80 \%$ in pigs and $84 \%$ in dogs [4]. This is not surprising as recent molecular studies indicate that $S$. scabiei in humans and animals are genetically distinct and that interbreeding or cross-infection appears to be extremely rare $[5,6]$.

It has been reported that, after 24 hours of exposure to an affected animal, household members develop selfclearing pruritic lesions primarily on the trunk, forearms, and thighs [7]. Differing host immune responses and disease etiology caused by related mite species may be a result of variable expression levels of homologous genes in different strains of S. scabiei, as well as the presence or absence of particular genes. Furthermore, whole-mite extracts contain a heterogeneous mixture of host and parasite antigens and vary in composition, potency, and purity. S. scabiei hominis cDNA sequence libraries [8] now provide a vast new store of information to target the generation of novel products for specific and sensitive diagnostic assays. Recombinant antigens promise a continuous, reproducible quantity of allergenic proteins in a purified form suitable for use in an in vitro assay.

Crusted, or Norwegian, scabies is rare and results from hyper-infestation with $S$. scabiei. A clinical and immunologic study of 78 prospectively identified patients over a 10-year period from northern Australia identified immunosuppression as a risk factor in over half [9]. Eosinophilia 
and elevated IgE levels occurred in $58 \%$ and $96 \%$ of patients respectively, with median IgE levels at 17 times the upper-limit of normal. A tendency to mount a Th2 response may underlie the apparent predisposition to develop crusted scabies. Preliminary studies on cytokine

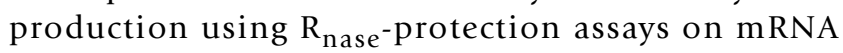
obtained from fresh peripheral blood mononuclear cells collected from patients with crusted scabies and uninfested controls, demonstrated a statistically significant elevation of interleukin (IL)-4 in crusted scabies [10••]. Treatment protocols, consisting of topical scabicides and keratolytic therapy with multiple doses of systemic ivermectin, have been associated with decreased mortality. New directions include the development of immunotherapy, as successfully used for house dust mite allergy, using purified, recombinant $S$. scabiei allergens to characterize and modulate the immune aberration responsible for crusted scabies.

\section{Chemotherapy}

In endemic situations, empirical treatment is often more cost effective than laboratory-based testing, and recently mass intervention programs with ivermectin in Africa [11], Brazil [12], and the Solomon Islands [13] have resulted in dramatic reductions in the prevalence of onchocerciasis, filariasis, intestinal helminths, and scabies. Approximately 250 million doses of ivermectin have been distributed over 15 years for the treatment of onchocerciasis in a variety of African countries, and it appears to be a safe, highly-effective treatment with few adverse events reported. Although ivermectin may be a suitable substitute for topical treatment of scabies in resource-poor communities where polyparasitism is common, mass treatment in endemic communities also creates an environment for emerging tolerance or resistance. Documentation of resistance against anti-ectoparasitic compounds is increasing. Clinical and in vitro evidence of ivermectin resistance has been documented in two patients with multiple recurrences of crusted scabies who had received between 35 and 58 doses of ivermectin over 4 to 4.5 years [14]. Experience with both acarid and insect pests, as demonstrated in human head lice, shows that management is difficult once drug resistance is established. There is an urgent need to develop better tools to monitor for drug resistance in order to monitor and define the extent of resistance in scabies mites. Videodermatoscopy has lately been described as a successful method for in vivo monitoring of drug effectiveness, with observations post treatment demonstrating the reduced-migration ability and eventual death of the mite under the skin [15]. However, continued epidemiologic studies and the development of molecular tools will identify the factors which govern the emergence and spread of resistant strains of S. scabiei. Alternate strategies include the detection of novel therapies, and recent studies suggest tea tree oil or other essential oils, via constitutive oxygenated terpenoids, may play a role as potential new acaricides $[16,17]$.

\section{Recombinant DNA technology applications in scabies research}

There is currently no animal model or in vitro culture system for propagating S. scabiei hominis available. This has significantly restricted research on the biology of the mite but recently has been overcome by the construction of S. scabiei hominis and S. scabiei vulpes cDNA libraries and Expressed Sequence Tag databases $[18,19]$. These now provide a vast new store of information to target the generation of novel products for diagnostic assays, chemotherapy, vaccine development, and drug targets. A number of scabies-mite homologues of house dust mite allergens have now been cloned [20$22]$, and with the identification of many more novel molecules imminent, fascinating and unexpected molecular discoveries will soon emerge. Parasite secretions and hidden antigens are recognized as playing key roles in stimulating and modulating host tissue responses, and the discovery of new S. scabiei gene families and their products can only further provide interesting insights into molecular biology and host immune evasion strategies of the scabies mite.

\section{Pediculosis}

Human lice are blood-sucking insects that infest the head, Pediculus humanus capitis; the body, P. humanus corporis; and the pubic region, Phthirus pubis. Diagnosis is primarily by the identification of live lice and/or viable eggs and the clinical symptom of intense pruritus. Human head lice is a major problem for children aged 3 to 12 years in both industrialized and developing countries, and it is currently estimated between 6 to 12 million head lice infestations occur in the United States annually [23].

The infestation is clearly more common in children, but head lice occur to an important degree in adults as well [24•]. This is of practical importance, as control strategies usually focus on treatment of school-aged children, neglecting the family contacts.

Strong evidence now exists for pediculicide resistance in many countries, with resistance reported to vary among countries, regions, and chemicals. Significant direct and indirect costs are associated with treatment, and anecdotal reports estimate hundreds to millions of dollars annually are spent on treatments. Treatment failure can now no longer be assumed to be a result of poor compliance or reinfestation, and often the lack of regional monitoring systems means parents are hindered in making informed decisions regarding the best treatment $[25 \bullet \bullet]$. Traditional, ad hoc, mass, school-based screening may not be the best use of resources to control infestations [26]. Instead, more accurate diagnosis of head lice infestation may provide the most effective means of controlling the escalating problem and ensuring proper use of pediculicides to counteract resistance. 


\section{Novel insecticides}

As the prevalence of resistant head lice increases worldwide, the need for novel forms of treatment to control pediculosis has intensified and is evidenced in the literature. Essential oils merit further study as potential pediculicides and have been the recent subject of a number of investigations $[27,28]$, including the efficacy of a slow-release citronella formulation as a repellent against head lice [29]. Studies indicated eucalyptus, marjoram, pennyroyal, and rosemary oils were more active than delta-phenothrin and pyrethrum controls on the basis of in vitro LT50 assays. This appeared largely as a result of action in the vapor phase. To date, none of these products have been evaluated in vivo for efficacy or toxicology by regulatory agencies. Essential oils are complex mixtures of compounds, many of which are monoterpenes, and two major components of tea tree oil, 1,8-cineole and terpinen-4ol, have recently been investigated and shown to cause inhibition of acetylcholinesterase activity, a common mechanism used by other pediculicides [30]. However, many chemical companies are unwilling to pursue potential new pesticides due to the high costs associated with development and registration. As a result, families are often trying untested home remedies with generally ineffective outcomes [31]. Recently, as for scabies, oral ivermectin has been identified as an effective pediculicide in polyparasitized communities [12]. However, the long term effects of using ivermectin for treatment of head lice are still not known.

\section{Recombinant DNA technology applications}

As for scabies, studies on the biology and host-parasite interactions of $P$. capitis, $P$. humanus, and $P$. pubis were relatively restricted due to limited genomic information. Recently, a number of cDNA libraries of the human body louse have been constructed (K. Fischer, unpublished information) [32,33]. Identified protein homologues described to date include defensin, two cysteine and five serine proteases, trypsin, glutathione-s-transferases, and cytochrome P450 genes. Again, the discovery of human lice gene families and their products will enable significant advances on host-parasite interactions and provide important insights into potential drug targets, chemotherapy, and vaccine candidates.

Other molecular studies have identified that the female bias commonly reported in head lice populations may be a result of lice carrying the endosymbiotic bacteria Wolbachia pipientis, a commonly known intracellular sex ratio distorter [34]. In nematodes, antibiotics targeted at the bacteria eventually result in host death, and thus antibiotic therapy has been heralded as a novel treatment for filariasis $[35,36]$. Antibiotics have been previously documented as having a lethal effect on lice [37] and may warrant further investigation as to an alternative treatment.

\section{Tungiasis}

Tungiasis is an ectoparasitic infestation caused by the penetration of the female sand flea Tunga penetrans into the skin. Both males and females are blood-feeding, but only the female flea penetrates permanently into the host [38]. Immediately after penetration, the flea begins an impressing hypertrophy. At the end, the abdomen has enlarged to about $1 \mathrm{~cm}$.

Typically, T. penetrans affects the periungual area of the toes, the heels, and the soles. However, embedded sand fleas can be found on almost every part of the body, such as the hands, elbows, neck, buttocks, and the genital region. Severe infestations with hundreds of embedded sand fleas are not rare. Although tungiasis is a self-limited infestation, complications are common in the endemic area. Sequels include deformation and loss of toenails, as well as deformation of digits. Superinfected lesions lead to formation of pustules, suppuration and ulcers. S. aureus and streptococci are frequently isolated, but other aerobic and anaerobic bacteria (such as clostridiae) are also found [39].

\section{Epidemiology and control}

Tungiasis occurs on the American continent from Mexico to northern Argentina, on several Caribbean islands, as well as in sub-Saharan Africa [40••]. Some years ago, an autochthonous case of tungiasis was reported from southern Italy [41]. It remains to be seen whether global warming and increased international travel will allow $T$. penetrans to establish its life cycle in areas where it has not been observed before in the future.

Prevalence and parasite burden are correlated, and in the endemic area, individuals harbor dozens-even hundreds-of fleas. Occurrence of tungiasis is not limited to sandy soils; it may also be found in banana plantations and in the tropical rain forest with different types of soil.

Clearly, tungiasis is a poverty-associated disease. A study in a fishing community in Brazil indicated that poor housing is the most important independent risk factor for heavy infestation [42].

Domestic animals such as dogs, cats, and pigs are important reservoirs. In a slum in Fortaleza (Northeast Brazil), $67 \%$ of dogs, and $50 \%$ of cats were found to be infested. Additionally, rodents may contribute to high attack rates. In one study, $59 \%$ of captured rats were found to be infested [43].

Due to the presence of a variety of domestic and sylvatic animals acting as reservoirs, control of tungiasis is difficult to achieve. Additionally, eggs, larvae, and pupae of T. penetrans may survive in the environment for several months. Consequently, the reduction of the human and animal reservoir without elimination of developmental stages in the environment would result in rapid reinfection. Surface spraying with insecticides has claimed to be effective in killing off-host stages of the sand flea, but there are no controlled studies to confirm this assumption. Environmental factors that govern the off-host life cycle of the flea are not understood.

Programs aiming at the control of this ectoparasite must also take into account the seasonal pattern of the disease. A recent longitudinal study has shown a seasonal 
variation of tungiasis infestation with a low incidence during the rainy season and high incidence and subsequent morbidity during the dry season [44].

\section{Diagnosis and therapy}

The patient typically complains about local itching, pain and the sensation of a foreign body at the site of penetration. Patients commonly report having visited beaches or farms. The diagnosis of tungiasis is made clinically. A biopsy of the lesion and histopathologic examination is not indicated. The natural history of tungiasis was described recently that divided it into five stages (Fortaleza Classification) $[45 \bullet \bullet]$.

The standard treatment is the surgical extraction of the flea under sterile conditions. There is no drug on the market with satisfactory clinical efficacy. Clinical trials performed in the last 2 years did not reveal any promising compound. A recent, randomized, controlled trial with oral ivermectin at a relatively high dose $(2 \times 300 \mu \mathrm{g} / \mathrm{kg}$ body weight $)$ did not show a significant efficacy [46]. Another trial reported some efficacy of topical ivermectin, metrifonate, and thiabendazole compared to a topical placebo lotion [47]. A pilot trial with silicone oil of extreme low viscosity indicated that embedded fleas are killed after the application of the oil on top of the rear cone of the parasite (Heukelbach, unpublished observation).

However, it can be questioned if chemotherapy after penetration of the flea makes any sense in the endemic area. Two studies testing repellents that are applied on the feet to reduce infestation showed very promising results; a case series, using a natural repellent based on coconut oil over 2 weeks, resulted in an impressive regression of clinical pathology in patients who were severely infested [48]. The twice-daily application of this plant-based repellent reduced the infestation rate in an urban slum in Brazil, where transmission rates are extremely high, by almost 90\% (Feldmeier et al., unpublished observation). In severely affected communities, the use of an effective repellent probably is a better approach to reduce morbidity than treatment after infestation.

\section{Future prospects}

Recently published studies identified Wolbachia bacteria in large numbers in T. penetrans from Africa and Latin America [49]. Possibly, Wolbachia of T. penetrans are obligatory endosymbionts similar to those described from other parasites such as filariae and nematodes. The role of Wolbachia in the biology of $T$. penetrans and in the pathogenesis of tungiasis is not known, but it is possible that Wolbachia endobacteriae of sand fleas contribute to the severe inflammation constantly surrounding embedded fleas. It needs also to be determined if Wolbachia bacteria enter the bloodstream in individuals infested with $T$. penetrans, causing systemic pathology.
A model has been established recently to infest laboratory-raised Wistar rats artificially with T. penetrans [50]. In a first study, the cytokine kinetics after infestation of Wistar rats has been described. The serum concentration of IL- 4 increased 2 days after penetration, and tumor necrosis factor (TNF)- $\alpha$ and IL- $1 \beta$ after 10 to 13 days [50]. Another study conducted in humans indicates that tungiasis induces an immune response in the human host characterized by an increase of the cytokines interferon- $\gamma$, TNF- $\alpha$ and IL-4 in the circulation [51].

Tungiasis in the Wistar rat can become a model to study basic mechanisms of local inflammation in the skin and immune response of the host. The model will also help to find pharmacologic substances able to interrupt the development of severe inflammation. Anti-inflammatory and antiparasiticidal compounds can now be tested under laboratory conditions. The use of the Wistar rats will also open new horizons for the establishment of the complete life cycle in the laboratory and will help to disentangle unknown biological aspects of the parasite.

A new tunga species, T. trimamillata, has been described recently in Ecuador [52]. Interestingly, this species also parasitizes humans. It is possible that, in the Andean region, this sand flea has a similar importance in resourcepoor communities as T. penetrans in the South American lowlands. As suggested by the molecular analysis of highlyconserved internal transcribed spacersequences, it is feasible that other hitherto not described species of Tunga coexist in the same area [53].

Tungiasis is still one of the least understood parasitic skin diseases. The habitat of off-host stages of the flea and the susceptibility of these stages to insecticides is not known. The epidemiology of the disease in endemic populations remains enigmatic. The mechanisms of the severe inflammatory reaction around lesions are not understood. These lacunae make effective control almost impossible at present.

\section{Cutaneous Larva Migrans}

Cutaneous larva migrans is a parasitic skin infestation caused by the accidental penetration of the larvae of animal hookworms (most commonly Ancylostoma braziliense) into the epidermis of humans. As humans are a parathenic host, larvae cannot develop; therefore, they migrate in the epidermis and eventually die. Thus, the infestation is self-limiting.

Infestation can take place anywhere skin comes into contact with soil which is contaminated by animal feces, such as beaches, sand boxes, or construction sites. Infestation may also occur via fomites, such as towels or clothes that came into contact with soil while drying.

The condition is extremely pruritic and uncomfortable. In a resource-poor community in northeast Brazil, about one third of patients complained of insomnia due to itch- 
ing [54•]. Secondary infection is common, especially in economically disadvantaged settings.

\section{Epidemiology}

Cutaneous larva migrans is common in tropical and subtropical regions throughout the world. Sporadic cases occur also in temperate zones. Sound population-based data are rare, and epidemiologic clues mainly come from tourists returning from holidays in the tropics.

In endemic areas, the great majority of dogs and cats are infected with hookworms. In a warm and humid environment, larvae can survive for several months when protected from direct sunlight and desiccation. Consequently, in resource-poor populations, prevalence of creeping eruption may be as high as $3 \%$ in the general population [54•], and incidence may be up to 1600 cases per 10,000 individuals per year (Jackson et al., unpublished data).

A recent longitudinal study conducted in an endemic area in Brazil has shown a characteristic seasonal variation, with most cases occurring during the rainy season; the point prevalence varied between $0.2 \%$ in the dry and $3.1 \%$ in the rainy season $[54 \bullet]$.

Risk factors for cutaneous larva migrans have never been studied appropriately. Preliminary data indicate that the occurrence is highly clustered in few households of a community (Jackson et al., unpublished observation).

\section{Diagnosis}

The characteristic clinical picture is a pruritic and serpiginous track. The infestation is diagnosed by the naked eye. Laboratory investigations are not helpful. Eosinophilia may be present.

Lesions are usually located on the feet, buttocks, and thighs but are also found on the arms, elbows, legs, knees, and back. They may occur at any topographic site including the face, the oral cavity, and the genitals $[54 \bullet]$.

Interestingly, the topographic distribution of lesions seems to be different between people living in resourcepoor, endemic communities and tourists. In a study of people living in an urban slum in the endemic area, most tracks occurred on the trunk and the legs, and not a single track was found on the feet; in $9 \%$ of lesions, tracks were diagnosed on the head [55]. This was explained by a different risk behavior of tourists and of inhabitants of deprived communities. Walking barefoot on beaches is a typical habit of tourists. In fact, walking barefoot to and on the beach has been the only risk factor identified so far for cutaneous larva migrans in a group of tourists [56]. In contrast, even in poor communities, people normally wear flip-flops at least. Regarding the people living in an urban slum, clothes probably serve as fomites. Clothes are hung up for drying on walls or fences-or put on the ground-and accidentally can be infested by crawling larvae.

\section{Treatment and prevention}

Some randomized trials have shown a high efficacy of oral ivermectin and oral albendazole [57]. A single dose of oral ivermectin $(200-300 \mu \mathrm{g} / \mathrm{kg})$ kills the larva. Oral albendazole $(400 \mathrm{mg} / \mathrm{d}$ for 3 days) shows excellent cure rates also. Thiabendazole ointment (10\%-15\%) applied to affected areas three times daily for 7 days seems to be as effective as the oral treatment.

To prevent infestation, animals should be banned from beaches. Unprotected skin should never come into contact with possibly contaminated ground. This means it is necessary to wear shoes and to refrain from lying directly on the sand at beaches or on greens to which dogs or cats have access. Lying on towels does not provide sufficient protection. Towels and clothes should not touch the ground when hung up for drying as they may serve as fomites.

\section{Outlook}

It is obvious that cutaneous larva migrans is a poverty-associated disease and that factors such as overcrowding, lack of sanitation, and poor health education contribute to high attack rates in humans. The prevalence of hookworm infection in domestic animals and the prevalence of cutaneous larva migrans in humans are linked. Identifying the exact factors which determine human attack rates in an endemic community requires comprehensive investigation of the animal reservoirs.

\section{Myiasis}

Myiasis is the infestation of the skin by larvae (maggots) of a variety of fly species (order Diptera), which for a certain period of time feed upon living, necrotic, or dead issue, liquid body substances, or the ingested food of their host. The entomologic classification of the responsible fly requires extensive entomologic knowledge and remains the domain of the Diptera specialist.

Schematically, myiasis can be divided into infestation of the skin, of natural cavities and orifices such as the ear and the vagina, and of mucous membranes. Skin myiasis is further differentiated into furuncular (sometimes called "hypodermic"), creeping (migratory), and wound (traumatic) myiasis. In furuncular and migratory myiasis, larvae penetrate into the subcutaneous tissue. In wound myiasis, flies deposit larvae in a suppurating wound, chronic ulcers, necrotic, or decomposing flesh.

As no systematic studies have been undertaken, data on prevalence and incidence in endemic areas do not exist.

\section{Disease manifestations}

Myiasis is self-limiting with the vast majority of cases showing minimal clinical pathology. However, particularly in myiases with a long duration of larval development, patients may suffer considerably. Feeding and growing larvae may cause severe inflammation and excruciating pain (eg, if located near or in the eye), resulting in distur- 
bance of sleep and mood. Feeling something moving under one's skin or on the top of a mucous membrane is a rather strange experience and explains why patients are extremely distressed. Moreover, some larvae species bury deeply into living tissue and feed gregariously resulting into considerable damage and disfigurement, especially if cavities of the head are attacked. Under very rare circumstances, larvae may migrate from the external eye via the orbita into the brain eventually leading to the death of the patient (infiltrative myiasis). Hence, myiasis can be considered a nuisance in no case.

\section{Diagnosis}

For the physician working in an endemic area, myiasis is commonly a first-look diagnosis. Patients outside the tropics are frequently referred from the general practitioner to specialists. According to the topographic localization and the clinical manifestation, myiasis can mimic many diseases of infectious or noninfectious origin. Breast myiasis was recently diagnosed by mammography [58]. Doppler ultrasound was found useful in the diagnosis of furuncular myiasis [59]. Laboratory investigations are not helpful.

\section{Immune response}

Although myiases cause considerable damage in livestock, immunologic parasite-host-interactions have been addressed only recently [60]. Obviously, immune responses depend on the duration and anatomic site of parasitism, the type of antigens released and the immunocompetence of the host. For example, blowflies (Calliphoridae) and flesh flies (Sarcophagidae) cause myiases of relatively short duration, as larvae mature within 4 to 7 days, offering few opportunities for an immune response to develop during primary infestation. In contrast, botflies (Oestridae) are harbored and feed for several weeks to several months. Here, the immune system has ample time to react.

Embedded fly larvae elicit both nonspecific-innate and

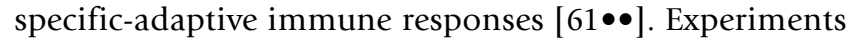
in various fly-host-models have shown that innate immunity is the result of the activation of natural killer cells, eosinophils, mast cells, and neutrophils. The interaction between larval antigens and major histocompatibility complex-II molecules on antigen-presenting cells induces adaptive immune responses, the nature of which varies according to parasite and animal species [60]. There is convincing evidence that larvae are able to downregulate immune responses of their host $[61 \bullet \bullet]$.

Thus far, the immune response has been studied in only one patient with myiasis. It was shown that eosinophils and $\mathrm{CD}^{+}{ }^{+} \mathrm{T}$ cells predominated in the inflammatory infiltrate surrounding an embedded larva. Immunohistochemic staining of the lesion indicated the presence of a unique effector cell population bearing the characteristics of cytotoxic $\mathrm{CD}^{+} \mathrm{T}$ cells but producing cytokines of the
Th2 pattern [62]. This puzzling observation awaits confirmation in a representative group of patients.

\section{Therapy}

Therapy has to be adapted to the type of myiasis. In myiasis affecting cavities and orifices (particularly the mouth, nose, and eye), all larvae have to be removed surgically. Wound myiasis requires complete debridement. Occlusion and suffocation approaches include petroleum jelly, liquid paraffin, beeswax, nail polish, or oil of high viscosity placed over the central punctum. Application of lard or a strip of bacon taped over the site also coaxes the larva to emerge to the skin surface. In addition, suffocation forces the larvae to reposition into an upward position so that it can be grabbed with tweezers or forceps.

A novel approach is the use of ivermectin, a broad anthelminthic with insecticidal properties. Several authors have successfully used oral and topical ivermectin in furuncular as well as cavity myiasis $[63,64]$.

\section{New prospects}

For centuries, clinicians have observed that certain fly larvae provide debridement of necrotic wounds, particularly of those occurring during military operations. Recently, a few centers systematically use larvae for debridement of chronic non-healing wounds and have coined the term "biosurgery" for this approach. It is clear that the crucial problem of biosurgery is a sepsis of the larvae. Nuesch et al. [65] reported superinfection in $21 \%$ of patients with biosurgery until the fly species was changed and a new method of disinfection was used. The future will show whether debridement with larvae becomes a safe and cost effective treatment for patients with necrotizing wounds or chronic ulcers.

A better understanding of the biology and the parasitehost-relationships of Calliphoridae and Sarcophagidae flies has provided the foundations for the development of a new discipline within forensic medicine. Forensic entomology is based on the assumption that larvae of these families feed and develop on carrion and that, by determining the degree of development of larvae together with environmental factors (temperature, humidity, etc.), the time of death can be precisely estimated. Identification of the many different species and their developmental stage is greatly facilitated by the rapidly expanding sequencing of

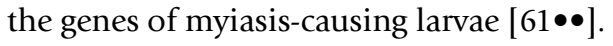

In veterinary medicine for the last couple of years, myiasis is identified by immunodiagnosis. This makes sense as domestic animals are repeatedly infested and parasitologic diagnosis sometimes is difficult. Whether immunodiagnosis or "bedside" polymerase chain reaction will replace clinical diagnosis in humans remains to be seen.

Given the observation that fly larvae exposed to infected brain are able to transmit scrapie to hamsters, it has been recently hypothesized that Hypoderma species. can act as vectors for animal prion diseases. 


\section{Conclusions}

The control of ectoparasitic infestations in resource-poor communities remains a challenge. Sound epidemiologic data, on which control measures in different settings could be based, are not at hand. Resistance of scabies mites and head lice to currently used antiparasiticidal compounds can be expected to increase in the next few years. This will hinder effective control further. The development of new compounds based on plant extracts could fill this therapeutic gap. In the case of tungiasis, the presence of Wolbachia in sand fleas offers new targets for therapy and control.

Molecular approaches, although in their infancy, are now providing a better understanding of the biology of ectoparasites and will become cornerstones for prevention and control in the future. Combining the existing knowledge on off-host development together with new molecular techniques will improve our understanding of parasitic skin diseases and may open new avenues for chemotherapy and vaccination.

\section{References and Recommended Reading}

Papers of particular interest, published recently, have been highlighted as:

- Of importance

- Of major importance

1. Hoy W, McDonald SP: Albuminuria: marker or target in indigenous populations. Kidney Int Suppl 2004, 66:25-31.

2. Arlian LG, Morgan MS, Estes SA, et al.: Circulating IgE in patients with ordinary and crusted scabies. J Med Entomol 2004, 41:74-77.

3. Lowenstein M, Kahlbacher H, Peschke R: On the substantial variation in serological responses in pigs to Sarcoptes scabiei var. suis using different commercially available indirect enzyme-linked immunosorbent assays. Parasitol Res 2004, 94:24-30.

4. Haas N, Wagemann B, Hermes B, et al.: Crossreacting IgC antibodies against fox mite antigens in human scabies. Arch Dermatol Res 2005, 296:327-331.

5. Walton S, Low Choy J, Bonson A, et al.: Genetically distinct dog-derived and human-derived Sarcoptes scabiei in scabiesendemic communities in northern Australia. Am J Trop Med Hyg 1999, 61:542-547.

6. Walton SF, Dougall A, Pizzutto S, et al.: Genetic epidemiology of Sarcoptes scabiei (Acari: Sarcoptidae) in northern Australia. Int J Parsitol 2004, 34:839-849.

7. Ambroise-Thomas P: Emerging parasite zoonoses: the role of host-parasite relationship. Int J Parasitol 2000, 30:1361-1367.

8. Harumal $\mathrm{P}$, Morgan MS, Walton SF, et al.: Identification of a homologue of a house dust mite allergen in a cDNA library from Sarcoptes scabiei var. hominis and evaluation of its vaccine potential in a rabbit/S. scabiei var. canis model. Am J Trop Med Hyg 2003, 68:54-60.

9. Roberts LJ, Huffam SE, Walton SF, Currie BJ: Crusted scabies: clinical and immunological findings in seventy-eight patients and a review of the literature. J Infect 2005, 50:375-381.

$10 . \bullet$ Walton SF, Holt DC: Scabies: new future for a neglected disease. Adv Parasitol 2004, 57:309-376.

This comprehensive review highlights recent advances in the study and control of scabies in human and veterinary medicine.

11. Tielsch JM, Beeche A: Impact of ivermectin on illness and disability associated with onchocerciasis. Trop Med Int Health 2004, 9:45-56
12. Heukelbach J, Winter B, Wilcke T, et al.: Selective mass treatment with ivermectin to control intestinal helminthiases and parasitic skin diseases in a severely affected population. Bull World Health Organ 2004, 82:563-571.

13. Lawrence G, Leafasia J, Sheridan J, et al.: Control of scabies, skin sores and haematuria in children in the Solomon Islands: another role for ivermectin. Bull World Health Organ 2005, 83:34-42.

14. Currie BJ, Harumal P, McKinnon M, Walton SF: First documentation of in vivo and in vitro ivermectin resistance in Sarcoptes scabiei. Clin Infect Dis 2004, 39:8-12.

15. Micali G, Lacarrubba F, Tedeschi A: Videodermatoscopy enhances the ability to monitor efficacy of scabies treatment and allows optimal timing of drug application. J Eur Acad Dermatol Venereol 2004, 18:153-154.

16. Morsy TA, Rahem MA, el-Sharkawy EM, Shatat MA: Eucalyptus globulus (camphor oil) against the zoonotic scabies, Sarcoptes scabiei. J Egypt Soc Parasitol 2003, 33:47-53.

17. Walton SF, McKinnon M, Pizzutto S, et al.: Acaricidal activity of Melaleuca alternifolia (Tea Tree) oil: in vitro sensitivity of Sarcoptes scabiei var. hominis to terpinen-4-ol. Arch Dermatol 2004, 140:563-566.

18. Fischer K, Holt DC, Harumal P, et al.: Generation and characterisation of cDNA clones from Sarcoptes scabiei var. hominis for an expressed sequence tag library: identification of homologues of house dust mite allergens. Am J Trop Med Hyg 2003, 68:61-64.

19. Ljunggren EL, Nilsson D, Mattsson JG: Expressed sequence tag analysis of Sarcoptes scabiei. Parasitology 2003, 127:139-145

20. Holt DC, Fischer K, Allen GE, et al.: Mechanisms for a novel immune evasion strategy in the scabies mite Sarcoptes scabiei: A multigene family of inactivated serine proteases. J Invest Dermatol 2003, 121:1419-1424.

21. Holt DC, Fischer K, Pizzutto SJ, et al:: A multigene family of inactivated cysteine proteases in Sarcoptes scabiei. J Invest Dermatol 2004, 123:240-241.

22. Pettersson EU, Ljunggren EL, Morrison DA, Mattsson JG: Functional analysis and localisation of a delta-class glutathione S-transferase from Sarcoptes scabiei. Int J Parasitol 2005, 35:39-48.

23. Hansen RC, O'Haver J: Economic considerations associated with Pediculus humanus capitis infestation. Clin Pediatr (Phila) 2004, 43:523-527.

24. Heukelbach J, Wilcke T, Winter B, Feldmeier H: Epidemiology and morbidity of scabies and pediculosis capitis in resourcepoor communities in northeast Brazil. Br J Dermatol 2005, 153:150-156

Epidemiologic data on scabies and pediculosis from longitudinal studies in resource-poor communities are presented in this article.

25. $\bullet$ Downs AM: Managing head lice in an era of increasing resistance to insecticides. Am J Clin Dermatol 2004, 5:169-177.

This article is a review of current pediculicides, resistance, and management of head lice.

26. Counahan M, Andrews R, Buttner P, et al.: Head lice prevalence in primary schools in Victoria, Australia. J Paediatr Child Health 2004, 40:616-619.

27. Yang YC, Lee HS, Clark JM, Ahn YJ: Insecticidal activity of plant essential oils against Pediculus humanus capitis (Anoplura: Pediculidae). J Med Entomol 2004, 41:699-704.

28. Yang YC, Choi HY, Choi WS, et al.: Ovicidal and adulticidal activity of Eucalyptus globulus leaf oil terpenoids against Pediculus humanus capitis (Anoplura: Pediculidae). J Agric Food Chem 2004, 52:2507-2511.

29. Mumcuoglu KY, Magdassi S, Miller J, et al.: Repellency of citronella for head lice: double-blind randomized trial of efficacy and safety. Isr Med Assoc J 2004, 6:756-759.

30. Mills C, Cleary BJ, Gilmer JF, Walsh JJ: Inhibition of acetylcholinesterase by Tea Tree oil. J Pharm Pharmacol 2004, 56:375-379.

31. Takano-Lee M, Edman JD, Mullens BA, Clark JM: Home remedies to control head lice: assessment of home remedies to control the human head louse, Pediculus humanus capitis (Anoplura: Pediculidae). J Pediatr Nurs 2004, 19:393-398. 
32. Pedra JH, Brandt A, Li HM, et al.: Transcriptome identification of putative genes involved in protein catabolism and innate immune response in human body louse (Pediculicidae: Pediculus humanus). Insect Biochem Mol Biol 2003, 33:1135-1143.

33. Kollien AH, Waniek PJ, Prols F, et al.: Cloning and characterization of a trypsin-encoding cDNA of the human body louse Pediculus humanus. Insect Mol Biol 2004, 13:9-18.

34. Perotti MA, Catala SS, Ormeno Adel V, et al.: The sex ratio distortion in the human head louse is conserved over time. BMC Genet 2004, 5:10.

35. Taylor MJ, Hoerauf A: A new approach to the treatment of filariasis. Curr Opin Infect Dis 2001, 14:727-731.

36. Hise AG, Gillette-Ferguson I, Pearlman E: The role of endosymbiotic Wolbachia bacteria in filarial disease. Cell Microbiol 2004, 6:97-104.

37. Shashindran CH, Gandhi IS, Krishnasamy S, Ghosh MN: Oral therapy of pediculosis capitis with cotrimoxazole. Br J Dermatol 1978, 98:699-700.

38. Witt LH, Linardi PM, Meckes $\mathrm{O}$, et al.: Blood-feeding of Tunga penetrans males. Med Vet Entomol 2004, 18:439-441.

39. Feldmeier $\mathrm{H}$, Heukelbach J, Eisele $\mathrm{M}$, et al.: Bacterial superinfection in human tungiasis. Trop Med Int Health 2002, 7:559-564.

$40 . \bullet$ Franck S, Feldmeier H, Heukelbach J: Tungiasis: more than an exotic nuisance. Travel Med Infect Dis 2003, 1:159-166.

This article provides a comprehensive review on all aspects of tungiasis.

41. Veraldi S, Carrera C, Schianchi R: Tungiasis has reached Europe. Dermatology 2000, 201:382.

42. Muehlen $\mathrm{M}$, Feldmeier $\mathrm{H}$, Wilcke $\mathrm{T}$, et al.: Identifying risk factors for tungiasis and heavy infestation in a resource-poor community in Northeast Brazil. Trans Roy Soc Trop Med Hyg 2005, In press.

43. Heukelbach J, Costa AML, Wilcke T, et al.: The animal reservoir of Tunga penetrans in severely affected communities of north-east Brazil. Med Vet Entomol 2004, 18:329-335.

44. Heukelbach J, Wilcke T, Harms G, Feldmeier H: Seasonal variation of tungiasis in an endemic community. Am J Trop Med Hyg 2005, 72:145-149.

45.• Eisele M, Heukelbach J, van Marck E, et al.: Investigations on the biology, epidemiology, pathology and control of Tunga penetrans in Brazil: I. Natural history of tungiasis in man. Parasitol Res 2003, 90:87-99.

This review provides a detailed clinical and microscopic description of the natural history of tungiasis.

46. Heukelbach J, Franck S, Feldmeier H: Therapy of tungiasis: a double-blinded randomized controlled trial with oral ivermectin. Mem Inst Oswaldo Cruz 2004, 99:873-876.

47. Heukelbach J, Eisele M, Jackson A, Feldmeier H: Topical treatment of tungiasis: a randomized, controlled trial. Ann Trop Med Parasitol 2003, 97:743-749.

48. Schwalfenberg S, Witt LH, Kehr JD, et al:: Prevention of tungiasis using a biological repellent: a small case series. Ann Trop Med Parasitol 2004, 98:89-94.
49. Heukelbach J, Bonow I, Witt LH, et al.: High infection rate of Wolbachia endobacteria in the sand flea Tunga penetrans from Brazil. Acta Trop 2004, 92:225-230.

50. Feldmeier H, Witt LH, Schwalfenberg S, et al.: Investigations on the biology, epidemiology, pathology and control of Tunga penetrans in Brazil V. Cytokine concentrations in experimentally infected Wistar rats. Parasitol Res 2004, 94:371-376.

51. Feldmeier $\mathrm{H}$, Heukelbach J, Eisele $\mathrm{M}$, et al.: Investigations on the biology, epidemiology, pathology and control of Tunga penetrans in Brazil: III. Cytokine levels in peripheral blood of infected humans. Parasitol Res 2003, 91:298-303.

52. Pampiglione S, Trentini M, Fioravanti ML, Gustinelli A: Differential diagnosis between Tunga penetrans (L., 1758) and T. trimamillata Pampiglione et al., 2002 (Insecta, Siphonaptera), the two species of the genus Tunga parasitic in man. Parasite 2004, 11:51-57.

53. Vobis M, D'Haese J, Mehlhorn H, et al.: Molecular biological investigations of Brazilian Tunga sp. isolates from man, dogs, cats, pigs and rats. Parasitol Res 2005, 96:107-112.

54. Heukelbach J, Wilcke T, Meier A, et al.: A longitudinal study on cutaneous larva migrans in an impoverished Brazilian township. Travel Med Infect Dis 2003, 1:213-218.

This study provides evidence for a strong seasonal variation of cutaneous larva migrans.

55. Heukelbach J, Wilcke T, Feldmeier H: Cutaneous larva migrans (creeping eruption) in an urban slum in Brazil. Int J Dermatol 2004, 43:511-515.

56. Tremblay A, MacLean JD, Gyorkos T, Macpherson DW: Outbreak of cutaneous larva migrans in a group of travelers. Trop Med Int Health 2000, 5:330-334.

57. Caumes E: Treatment of cutaneous larva migrans and Toxocara infection. Fundam Clin Pharmacol 2003, 17:213-216.

58. de Barros N, D'Avila MS, de Pace BS, et al.: Cutaneous myiasis of the breast: mammographic and us features-report of five cases. Radiology 2001, 218:517-520.

59. Quintanilla-Cedillo MR, Leon-Urena H, Contreras-Ruiz J, Arenas R: The value of Doppler ultrasound in diagnosis in $\mathbf{2 5}$ cases of furunculoid myiasis. Int J Dermatol 2005, 44:34-37.

60. Otranto D: The immunology of myiasis: parasite survival and host defense strategies. Trends Parasitol 2001, 17:176-182.

$61 . \bullet$ Otranto D, Stevens JR: Molecular approaches to the study of myiasis-causing larvae. Int J Parasitol 2002, 32:1345-1360.

This is a comprehensive overview on the taxonomy, biology, and molecular characterization of fly larvae responsible for human and animal myiases.

62. Norwood C, Smith KJ, Neafie R, Skelton H: Are cutaneous reactions to fly larvae mediated by CD4+, TIA+ NK1.1 T Cells? J Cutan Med Surg 2001, 5:400-405.

63. Costa DC, de Tarso Ponte Pierre-Filho, Mac Cord Medina F, Mota RG, Carrera CR: Use of oral ivermectin in a patient with destructive rhino-orbital myiasis. Eye 2005, In press.

64. De Tarso P, Pierre-Filho P, Minguini N, et al.: Use of ivermectin in the treatment of orbital myiasis caused by Cochliomyia hominivorax. Scand J Infect Dis 2004, 36:503-505.

65. Nuesch R, Rahm G, Rudin W, et al.: Clustering of bloodstream infections during maggot debridement therapy using contaminated larvae of Protophormia terraenovae. Infection 2002, 30:306-309. 